\title{
Extensor muscle-preserving laminectomy in treating multilevel cervical spondylotic myelopathy compared with laminoplasty
}

\author{
Zhiming Yu ${ }^{1 \#}$, Da He ${ }^{2 \#}$, Jiachao Xiong ${ }^{1 \#}$, Zhimin Pan ${ }^{1,3}$, Lingxuan Feng ${ }^{1}$, Jiang Xu ${ }^{1}$, Zhimin Han ${ }^{4}$, \\ Cristian Gragnaniello ${ }^{5}$, Hisashi Koga ${ }^{6}$, Kevin Phan $^{7}$, Parisa Azimi ${ }^{8,9}$, Jong-Joo Lee ${ }^{10}$, Yoon Ha ${ }^{3}$, Kai Cao ${ }^{1}$ \\ ${ }^{1}$ Spine Center, the Second Affiliated Hospital of Nanchang University, Nanchang 330006, China; ${ }^{2}$ Department of Spine Surgery, Beijing Jishuitan \\ Hospital, Fourth Clinical College of Peking University, Beijing 100035, China; ${ }^{3}$ Department of Neurosurgery, Spine and Spinal Cord Institute, \\ Yonsei University College of Medicine, Seoul, Korea; ${ }^{4}$ Department of Orthopaedics, the First Affiliated Hospital of Nanchang University, Nanchang \\ 330006, China; ${ }^{5}$ Department of Neurosurgery, University of Illinois at Chicago, Chicago, IL, USA; ${ }^{6}$ Department of Orthopaedics, Iwai Orthopaedic \\ Medical Hospital, Tokyo, Japan; ${ }^{7}$ NeuroSpine Surgery Research Group (NSURG); Prince of Wales Clinical School, University of New South Wales, \\ Sydney, Australia; ${ }^{8}$ Neuroscience Research Center, ${ }^{9}$ Functional Neurosurgery Research Center, Shahid Beheshti University of Medical Sciences, \\ Tehran, Iran; ${ }^{10}$ Department of Neurosurgery, Bundang Jesaeng Hospital, Seongnam, Korea \\ Contributions: (I) Conception and design: K Cao, Y Ha; (II) Administrative support: K Cao, Spine Center, the Second Affiliated Hospital of Nanchang \\ University; (III) Provision of study materials or patients: K Cao, Z Yu; (IV) Collection and assembly of data: Z Pan, L Feng, J Xu, Z Han; (V) Data \\ analysis and interpretation: D He, J Xiong; (VI) Manuscript writing: All authors; (VII) Final approval of manuscript: All authors. \\ \#These authors contributed equally to this work. \\ Correspondence to: Kai Cao, MD, PhD. Spine Center, the Second Affiliated Hospital of Nanchang University, \#1 Minde Rd., Nanchang 330006, \\ China. Email: kaichaw@126.com; Yoon Ha, MD, PhD. Department of Neurosurgery, Spine and Spinal Cord Institute, Yonsei University College of \\ Medicine; 50 Yonsei-ro, Seodaemun-gu, Seoul 03722, Korea. Email: hayoon@yuhs.ac.
}

Background: Laminectomy and laminoplasty are popularly used in posterior cervical spine surgery but still have involved complications. We aimed to compare the clinical outcomes of microscope-assisted extensor muscle-preserving laminectomy (MA-EMPL) and open-door laminoplasty (ODLP) in treating multilevel cervical spondylotic myelopathy (MCSM).

Methods: A prospective study was designed to enroll twenty patients with MCSM underwent MA-EMPL, and recruit twenty-four patients with MCSM received ODLP (control). Radiographic measurements, outcome indicators including Japanese Orthopedic Association (JOA) score and visual analogue score (VAS) were used to evaluate technical effectiveness. Surgical complications were documented to assess technical safety.

Results: Postoperative cervical curvature index and range of neck motion (ROM) were not significantly changed except ROM in ODLP group. Postoperative JOA score and VAS in both groups showed improvements at final follow-up. There was no statistical difference in postoperative neurological recovery rates between two groups $(67.6 \% \pm 17.8 \%$ vs. $70.15 \% \pm 19.6 \%, \mathrm{P}=0.632)$. However, VAS was significantly lower at postoperative 1 month in MA-EMPL group compared with ODLP group $(\mathrm{P}<0.001)$. The incidences of C5 palsy were 0 vs. 16.7\% between MA-EMPL group and ODLP group. There was no axial symptom occurred in MA-EMPL group while six patients in ODLP group ( 0 vs. $25 \%, \mathrm{P}=0.049)$. In addition, the mean blood loss and hospital stay were lesser in MA-EMPL group compared with ODLP group $(\mathrm{P}<0.001, \mathrm{P}=0.002$, respectively).

Conclusions: MA-EMPL is an effective, safe and minimally invasive method in treatment of MCSM. Compared with ODLP, MA-EMPL has advantage to decrease intraoperative blood loss, hospital stay, postoperative VAS and axial symptom, as well as preserve postoperative ROM.

Keywords: Cervical spondylotic myelopathy (CSM); extensor muscles; laminectomy; laminoplasty; microscopeassisted surgery

Submitted Jun 26, 2019. Accepted for publication Jul 08, 2019.

doi: $10.21037 / \mathrm{atm} .2019 .08 .17$

View this article at: http://dx.doi.org/10.21037/atm.2019.08.17 


\section{Introduction}

Surgical strategy for cervical spondylotic myelopathy (CSM) has been a controversial issue in recent years (1). The operative schemes including approach and procedure for CSM depend on lesion site and involved segments in cervical spine. Anterior surgery mainly includes anterior cervical discectomy and fusion, anterior cervical corpectomy and fusion, and artificial cervical disc replacement. Posterior surgery generally includes laminectomy, laminoplasty or laminotomy. The anterior and posterior surgeries can achieve equivalent curative effects in treating CSM when strict indications chosen (2). However, complications and reoperation rate are higher in anterior surgeries although no significant difference in recovery rate of neurological function between the two approaches $(3,4)$. Anterior approach is commonly utilized for CSM lesions $\leq 2$ segments, while posterior approach is primarily considered in cases of multilevel CSM (MCSM) (5).

Of the posterior approaches, laminotomy is a partial removal of lamina and not optimal for treatment of MCSM. Although expansive cervical laminoplasty (ECLP) and conventional extensive laminectomy have been used to treat MCSM $(6,7)$, ECLP has the drawbacks such as surgery-related axial symptom and loss of cervical lordosis $(8,9)$, while conventional laminectomy is insufficient at saving range of neck motion (ROM) (7). Because posterior extensor muscles play a critical role in cervical dynamic stability (10). Inversely, stripping of these muscles can lead to surgical complications such as axial pain, cervical instability and even kyphosis $(9,11,12)$.

In order to minimize the stripping to cervical extensor muscles and decrease relevant complications, we advocated the microscope-assisted extensor muscle-preserving laminectomy (MA-EMPL) (12) to treat selected patients with MCSM. In this study, we aimed to evaluate this technical effectiveness and safety by analyzing its clinical outcomes and complications compared with laminoplasty.

\section{Methods}

\section{Case collection}

A prospective study was designed to draw the conclusion of this research. Twenty patients with MCSM (3-4 segments) had MA-EMPL and 24 patients with MCSM (3-4 segments) underwent open-door laminoplasty (ODLP) were analyzed from December 2013 to December 2016 (Table 1). Inclusion criteria for both groups were: (I) adult patient with CSM $\geq 3$ segments stenosis; (II) local cervical kyphosis $<10^{\circ}$; (III) spondylolisthesis $<3.5 \mathrm{~mm}$; (IV) occupying ratio of ossification of posterior longitudinal ligament to diameter of cervical canal $<50 \%$. Exclusion criteria for both groups were: cervical fracture/dislocation, cervical spinal deformity, main compression on anterior dural sac, and patient had severe radicular symptom $(12,13)$. The written informed consents were obtained from eligible patients and the study was designed in conformity with the Declaration of Helsinki.

\section{Variables analyses}

Relevant data were analyzed to compare the clinical outcomes between MA-EMPL group and ODLP group. To reduce outcome bias, radiographic parameters were measured by one radiologist and one clinical fellow and reported as average values. Cervical curvature (represented as $\mathrm{C}$ value) was measured by Borden's method (14) (Figure 1). ROM was recorded to compare cervical movements from pre- to postoperatively in both groups, and pre- and postoperative ROM change was calculated by measuring cervical extension/flexion radiograph (Figure 2).

Clinical outcomes were evaluated with Japanese Orthopaedic Association (JOA) score preoperatively, postoperative 3 and 12 months, and the progression at final follow-up (neurological recovery rate at postoperative 12 months) was calculated as (final JOA - preoperative JOA) $/(17$-preoperative JOA) $\times 100 \%$. The JOA score assesses cervical functionality, with higher score indicating better condition. Visual analogue score (VAS) was used to assess surgery-related pain. In addition, surgical complications including C5 palsy and axial symptom (AS) were documented to appraise therapeutic safety. Moreover, the intraoperative blood loss and hospital stay were recorded in all patients.

\section{Surgical procedures}

To decrease clinical heterogeneity, consistent surgical procedures were performed by an experienced senior surgeon in our department. Operative procedures in MAEMPL group was shown in Figures 3,4. The patient was placed in prone position with general anesthesia and her/his skull was fixed with a Mayfield holder. Under the surgical microscope, spinous process was split to base through the middle with a $1 \mathrm{~mm}$ drill after making a skin incision and an opening of the fascia along the midline (the muscles/ 
Table 1 Demographics and surgical parameters in two groups

\begin{tabular}{lccc}
\hline Variable & MA-EMPL group $(n=20)$ & ODPL group $(n=24)$ & 0.438 \\
\hline Age (years) & $59.8 \pm 10.5$ & $62.0 \pm 9.8$ & \\
Gender & & & 14.934 \\
Male & 11 & 10 & 0.733 \\
Female & 9 & $16.8 \pm 14.3$ & 0.786 \\
Duration of symptom (m) & $18.2 \pm 15.1$ & & 17 \\
Segment of CSM & 14 & 7 & $<0.001$ \\
3 segments & 6 & $239.8 \pm 127.2$ & 0.002 \\
4 segments & $73.6 \pm 41.4$ & $7.5 \pm 4.2$ & \\
Blood loss (mL) & $4.3 \pm 2.9$ & & \\
Hospital stay (days) & & & \\
\hline
\end{tabular}

$\mathrm{P}<0.05$ is considered statistically significant. CSM, cervical spondylotic myelopathy; MA-EMPL, microscope-assisted extensor musclepreserving laminectomy; ODPL, open-door laminoplasty.

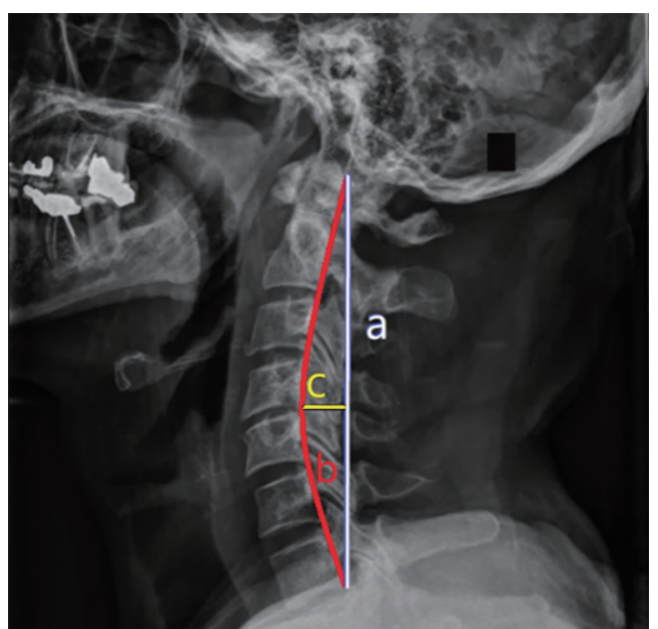

Figure 1 The schema for measuring cervical curvature. As shown on cervical lateral radiograph, "line a" starts from the posterosuperior point of C2's odontoid process to end at the posteroinferior point of $\mathrm{C} 7$ vertebra. A fitting curve (line b) draws along the posterior margin of $\mathrm{C} 2-7$ vertebrae. We define the longest distance from the posterior margin between $\mathrm{C} 4$ and $\mathrm{C} 5$ vertebral interval (on line b) perpendicular to line a as the cervical curvature (line c), which is presented as $\mathrm{C}$ value.

ligaments attaching on spinous process were preserved). A $3 \mathrm{~mm}$ drill was used to drill off the base of spinous process from lamina and a Gelpi single-tooth hook was used to distract and expose lamina. Under the microscope field, following polishing lamina with the width about $1.5 \mathrm{~cm}$, a Kerrison punch was used to remove the paper-like thin ventral lamina. Then, dural sac was revealed and hemostasis and surgical field clearing were performed through a bipolar coagulation. To achieve further expansion of spinal canal in multi-segment scenario, skipping performances were recommended. Removal of ligamentum flavum and undercutting of the inferior and superior of the skipped laminae were employed to make a "dome plasty". The procedures were repeated at the other stenosis segment. The final step is to close the splitted spinous processes and sutured the incision. ODLP was conducted as described in the original open-door technique (15).

\section{Perioperative care and follow-up}

All patients were followed up at least for 12 months (mean 19 months; range, 12-37 months). Preoperative cefazolin was administrated on the day of surgery, analgesia was routinely administrated for postoperative 2 days. Drainage was removed when the drainage amount was $\leq 50 \mathrm{~mL} / 24 \mathrm{~h}$. All patients had a soft cervical collar for a month after surgery. CT scans and MRI were taken to analyze postoperative radiographic outcomes.

\section{Statistical analysis}

All statistical analyses were performed by SPSS 19.0 statistical software (SPSS Inc., Chicago, USA). Data were presented as mean $\pm \mathrm{SD}$. A probability less than 0.05 was 

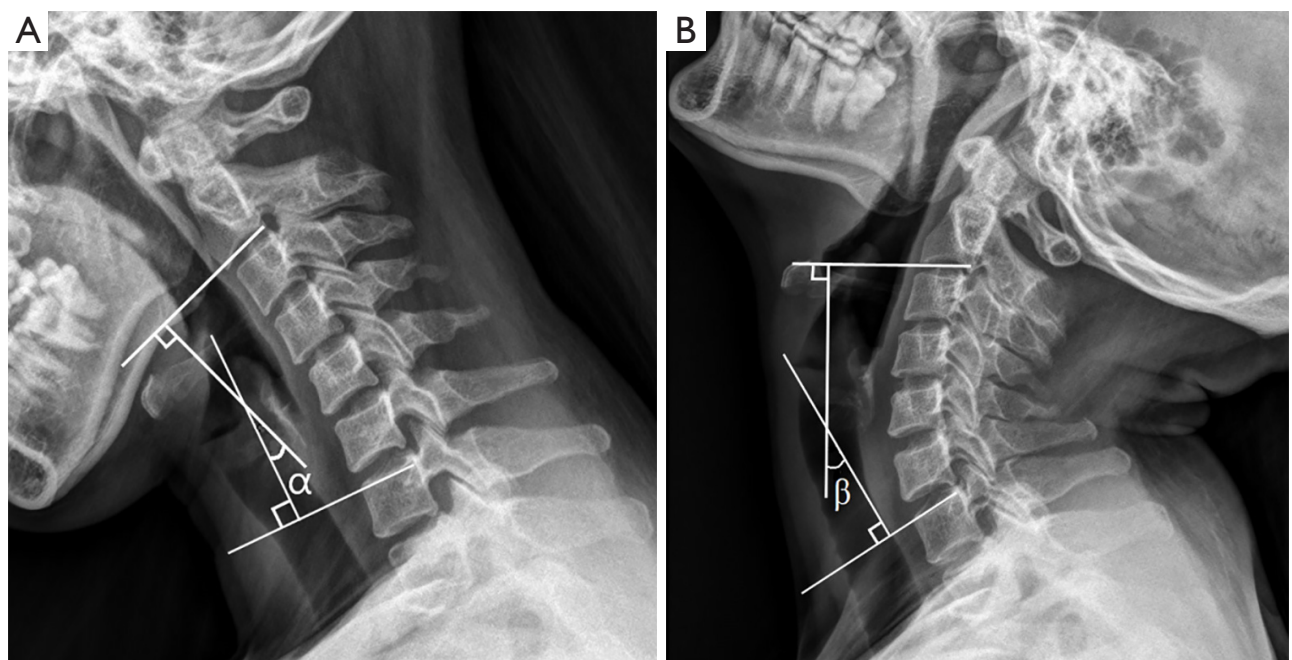

Figure $2 \mathrm{ROM}=$ flexion $\mathrm{C} 2-7 \mathrm{Cobb}$ angle $(\angle \alpha$ on Figure $2 A)+$ extension $\mathrm{C} 2-7 \mathrm{Cobb}$ angle $(\angle \beta$ on Figure $2 B)$. Retained $\mathrm{ROM}=$ postoperative ROM - preoperative ROM. ROM, range of neck motion.
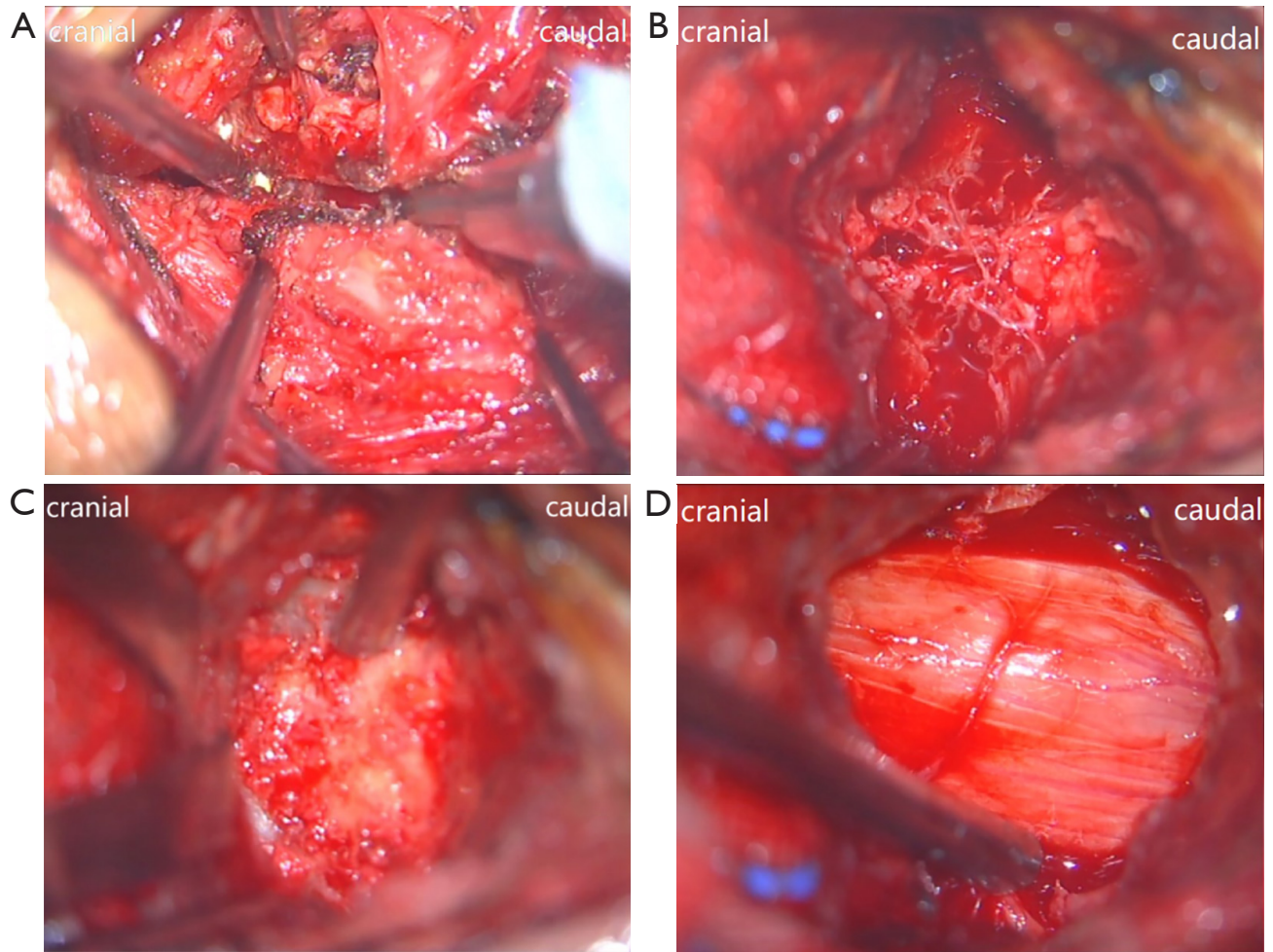

Figure 3 Intraoperative procedures. (A) Spinous process is longitudinally splitted by a 1-mm drill; (B) lamina was exposed after the base of spinous process drilled off from lamina; (C) the lamina was polished by drilling as thin as possible; (D) the dural sac is completely decompressed after removal of lamina, ligamentum flavum and "dome" plasty. 

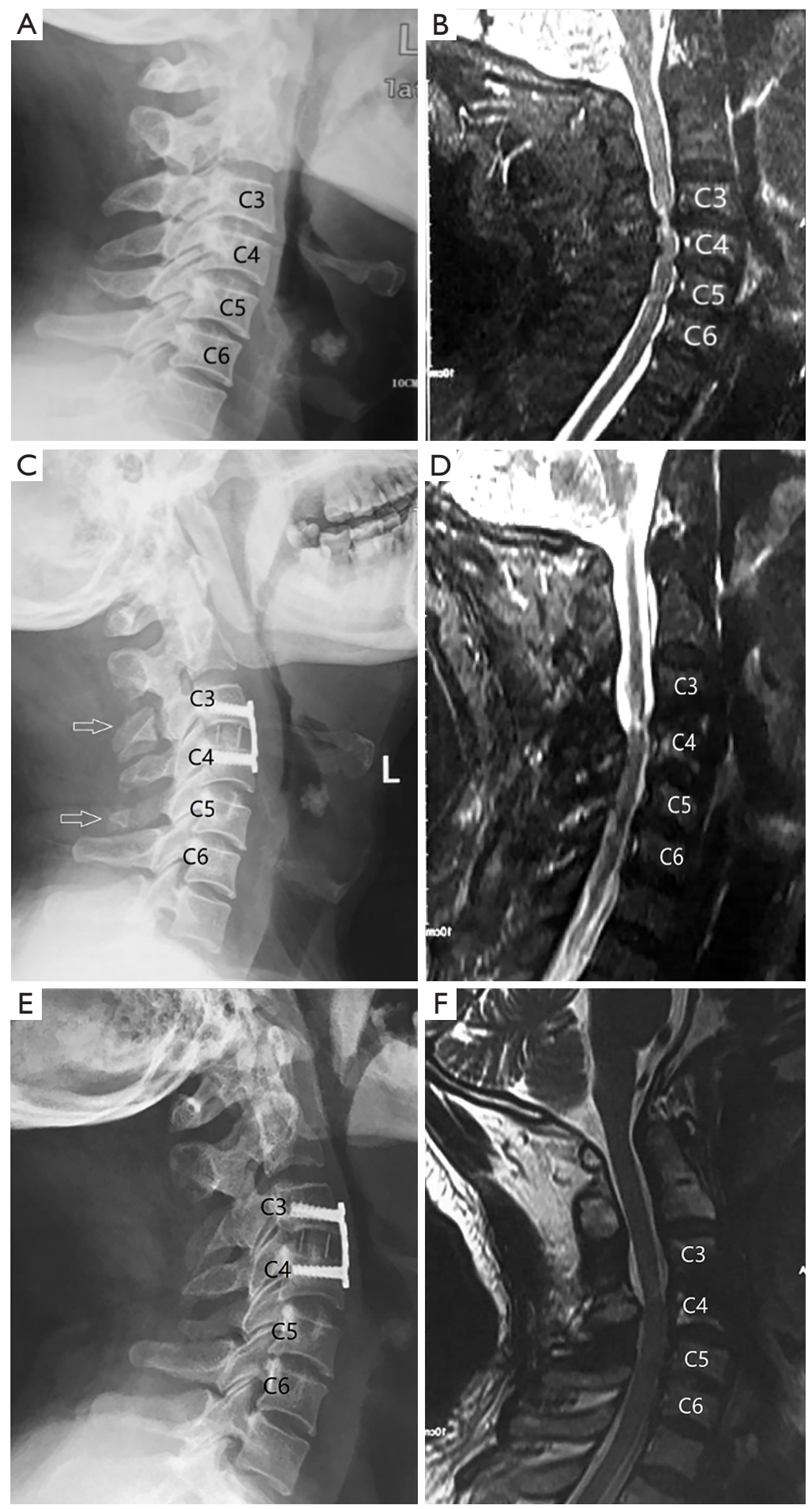

Figure 4 A 56-year-old male underwent a MA-EMPL. (A,B) The preoperative images show that MSCM occurs at C3 to C5, which segmental spinal cord was compressed; (C) the patient underwent an anterior cervical discectomy and fusion at C3/4 but his symptom did not relief well. Then he performed a MA-EMPL (arrows); (D) after the MA-EMPL, C3-5 spinal cord was decompressed, and the corresponding segmental extensor muscles were preserved; (E,F) the patient's cervical lordosis was achieved, spinal cord was decompressed, and extensor muscles were preserved at final follow-up. MSCM, multilevel cervical spondylotic myelopathy; MA-EMPL, microscopeassisted extensor muscles-preserving laminectomy. 
Table 2 Pre- and postoperative cervical curvature index (C value) and ROM

\begin{tabular}{|c|c|c|c|}
\hline Variable & MA-EMPL group & ODLP group & $\mathrm{P}$ \\
\hline \multicolumn{4}{|l|}{ C value (mm) } \\
\hline Pre-op & $10.8 \pm 3.04$ & $11.6 \pm 3.13$ & 0.354 \\
\hline Final follow-up & $9.75 \pm 2.95$ & $9.86 \pm 3.27$ & 0.899 \\
\hline$P$ & 0.212 & 0.056 & - \\
\hline Pre-op & $54.3 \pm 10.6$ & $55.1 \pm 12.4$ & 0.804 \\
\hline Final follow-up & $50.8 \pm 9.3$ & $46.7 \pm 11.8$ & 0.170 \\
\hline Retained ROM & $-3.6 \pm 4.2$ & $-8.3 \pm 5.0$ & $<0.001$ \\
\hline $\mathrm{P}$ & 0.212 & 0.016 & - \\
\hline
\end{tabular}

$\mathrm{P}<0.05$ is considered statistically significant. ROM, range of neck motion; MA-EMPL, microscope-assisted extensor muscle-preserving laminectomy; ODPL, open-door laminoplasty.

Table 3 Pre- and postoperative JOA score and VAS in two groups

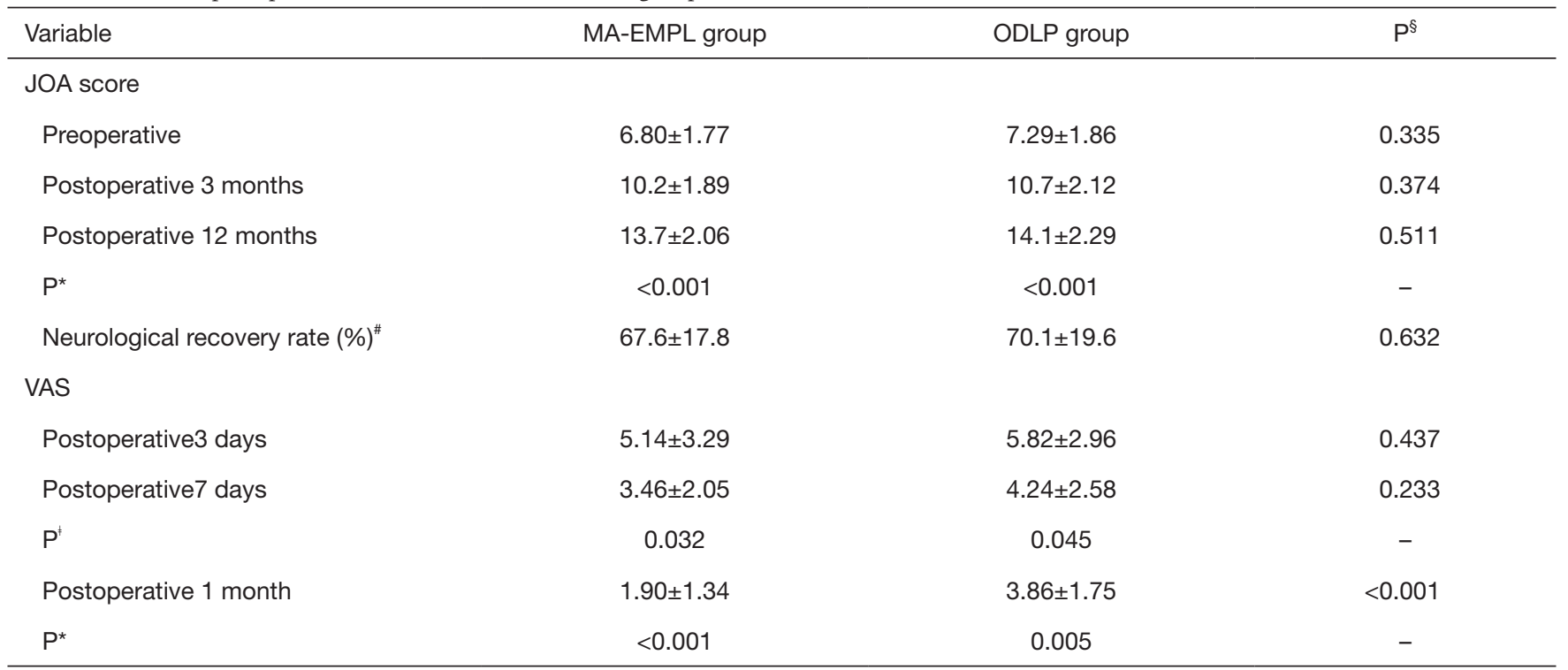

§, P for two groups; *, P for pre- and postoperative 12 months; ", neurological recovery rate was calculated by the aforementioned equation with the data at pre- and post-op 12 months; ${ }^{\ddagger}, \mathrm{P}$ for pre- and postoperative 10 days. $\mathrm{P}<0.05$ was considered statistically significant. JOA, Japanese Orthopaedic Association; VAS, visual analogue scale; MA-EMPL, microscope-assisted extensor muscle-preserving laminectomy; ODPL, open-door laminoplasty.

considered statistically significant. Preoperative and followup data were compared by paired $t$-test. Independentsample $t$-test was used to compare corresponding data between two groups.

\section{Results}

Patient demographic characteristics and surgical parameters are summarized in Table 1. There was no significant difference in the documented demographics. All patient incisions healed primarily. Most postoperative radiographic parameters were not significantly changed except the ROM in ODLP group (Table 2). JOA score and VAS showed improvements after surgery in both groups (Table 3). Complications occurred more in ODLP group (Table 4). In addition, the mean blood loss was significantly lesser in 
Table 4 Postoperative incidences of C5 palsy and AS

\begin{tabular}{lccc}
\hline Variable & MA-EMPL group & ODLP group & P \\
\hline Incidence of C5 palsy & $0 \%(0 / 20)$ & $16.7 \%(4 / 24)$ & 0.165 \\
Incidence of AS & $0 \%(0 / 20)$ & $25 \%(6 / 24)$ & 0.049 \\
\hline
\end{tabular}

$\mathrm{P}<0.05$ is considered statistically significant. AS, axial symptom; MA-EMPL, microscope-assisted extensor muscle-preserving laminectomy; ODPL, open-door laminoplasty.

MA-EMPL group compared with ODLP group $(73.6 \pm 41.4$ vs. $239.8 \pm 127.2 \mathrm{~mL}, \mathrm{P}<0.001)$, also the mean hospital stay was distinctively lesser in MA-EMPL group $(4.3 \pm 2.9 \mathrm{vs}$. $7.5 \pm 4.2$ days, $\mathrm{P}=0.002$ ).

\section{Radiographic measurements}

Medical images were used to indicate pre- and postoperative cervical morphologies and ROM. The preoperative radiographic data showed no significant difference between the two groups (Table 2). Postoperative cervical curvature indexes $(\mathrm{C}$ value) did not significantly change in both groups $(\mathrm{P}=0.212$ vs. $\mathrm{P}=0.056)$. The $\mathrm{ROM}$ was not significantly changed after surgery in MA-EMPL group but statistically decreased in ODLP group $(\mathrm{P}=0.212$ vs. $\mathrm{P}=0.016)$. Accordingly, the retained ROMs between two groups showed statistical difference $(\mathrm{P}<0.001)$.

\section{Neurofunctional outcomes and surgical complications}

Postoperative JOA score and VAS significantly improved in both groups (Table 3). There was no statistical difference in postoperative neurological recovery rates between two groups $(67.6 \% \pm 17.8 \%$ vs. $70.1 \% \pm 19.6 \%, \mathrm{P}=0.632)$. However, VAS was lower at postoperative 1 mon in MAEMPL group compared with ODLP group $(1.90 \pm 1.34$ vs. $3.86 \pm 1.75, \mathrm{P}<0.001)$, although it significantly reduced in both groups $(\mathrm{P}<0.001$ vs. $\mathrm{P}=0.005)$.

Surgical complications including axial symptom and C5 palsy were used to reveal the two technical safeties in treating MCSM (Table 4). The incidences of C5 palsy were 0 vs. $16.7 \%$ between MA-EMPL group and ODLP group $(\mathrm{P}=0.165)$. The axial symptom occurred in MA-EMPL group was significantly lesser than ODLP group $(\mathrm{P}=0.049)$. The patients' axial symptoms were alleviated after physical therapy.

\section{Discussion}

Laminectomy, laminoplasty and related modified techniques are widely accepted to treat CSM $(4,6,7,9,15-17)$. Based on our knowledges, imaging and clinical outcomes between ODLP and MA-EMPL after treating MCSM have rarely been compared in literature. With the both methods, we treated a series of MCSM patients and found that MAEMPL was an effective (saving normal cervical curvature and ROM, improving functional outcomes), safe (less complications) and minimally invasive (preserving extensor muscles) technique in treatment of MCSM. Compared with ODLP, MA-EMPL achieved similar neurofunctional outcomes but had the advantages in reducing intraoperative blood loss, hospital stay, postoperative complications and VAS, as well as preserving postoperative ROM.

\section{Radiographic outcomes}

In present study, ROM was retained in MA-EMPL group but decreased in ODLP group after surgery. Accordingly, postoperative retained ROMs were statistically different between the two groups $(\mathrm{P}<0.001)$. Bartels et al. compared pre- and post-operative ROM after laminoplasty and laminectomy in treating cervical spondylotic myelopathy, and found that there were no clinically important differences (18). The main reason for postoperative ROM retained well in MA-EMPL group but not in ODLP group at current study maybe because extensor muscles were well preserved in ODLP patients which were dynamically better to extend cervical spine after surgery. In addition, postoperative cervical curvature ( $\mathrm{C}$ value) did not significantly change in both groups which means cervical curvature could be maintained by the two techniques.

\section{Neurological results and complications}

JOA score is a clinical index used for assessment of cervical spinal cord function including ambulation, sensation and muscular tension (19). Literatures reported that ODLP could enlarge spinal canal and decompress intraspinal nervous tissue, which improved $45-80 \%$ neurological function (calculated by JOA scores) for patients with 
MCSM at postoperative 1-2 years $(20,21)$. In this study, JOA scores significantly improved after surgeries in both groups. In MA-EMPL group, we achieved adequate cervical spinal canal decompression with the aid of microscopic visual magnification to perform lamina removal, recess canal enlargement and "dome" plasty. These surgical procedures have the same effect as laminoplasty. Thus, postoperative neurological recovery rates were equally improved in both of MA-EMPL and ODLP groups $(\mathrm{P}=0.632)$, which were superior to Liu et al.'s study (13). In their study, the authors employed expansive open-door laminoplasty as well as laminectomy and instrumented fusion (LIF) to treat ossification of the posterior longitudinal ligament. They found that the neurological recovery rates were $46.3 \pm 15.8 \%$ in laminoplasty group and $52.0 \pm 15.3 \%$ in LIF group after surgeries. The superior neurological recovery rate after MA-EMPL in current study may owe to the thorough nerve decompression assisted by microscope.

In current study, VAS declined in postoperative 1 week in both groups but decreased more in MA-EMPL group at postoperative 1 month compared with ODLP group, although VAS reduced significantly at postoperative 1 mon in both groups. It indicated that postoperative pain relief in MCSM patients was more dramatic after treating by MA-EMPL compared with ODPL because obvious lesser invasion to cervical extensor muscles.

Axial symptoms include neck and shoulder pain especially cramps, and stiffness in cervical posterior extensor muscles, which mainly results from cervical posterior structures manipulation (22). Its incidence was significant higher in ODLP group of present study $(\mathrm{P}=0.049)$. Takeuchi et al. presumed that postoperative axial symptoms might be related to detachment or atrophy of posterior extensor musculature, which could result from disuse of neck function after laminoplasty (23).

Although posterior approaches have been widely used in the treatment of MCSM, some surgical complications including C5 palsy should not be ignored. C5 palsy is defined as a paresis of the deltoid or biceps brachii muscle. In Tsuji et al.'s study (24), they concluded that the larger postoperative space anterior to spinal cord was positively correlated with the higher incidence of C5 palsy. The incidences of C5 palsy were 0 vs. $16.7 \%$ between MAEMPL group and ODLP group in current study, to a certain extent, this result may indicate that laminoplasty tends to cause C5 palsy compared with MA-EMPL. Because spinal cord could shift more posteriorly after ODPL compared with MA-EMPL. In MA-EMPL group, the skipped and "domed" posterior arch stood in situ which limited spinal cord shifting posteriorly too much.

\section{The potential relation between intraoperative blood loss and hospital stay}

Generally, intraoperative blood loss is around $200-300 \mathrm{~mL}$ in laminoplasty (25-27), which is around $100-320 \mathrm{~mL}$ in laminectomy $(25,28)$. Blood loss in the ODLP group was significantly more than the MA-EMPL group due to more invasive procedures in present study $(\mathrm{P}<0.001)$. Another reason for lesser blood loss in MA-EMPL group was that the coagulation was easier and more thorough in the enhanced visual field under the microscope. In addition, hospital stay was significant shorter in the MA-EMPL group compared with the ODLP group due to less invasive surgery in the former too $(\mathrm{P}=0.002)$. Basically, there was no drainage after surgery in MA-EMPL group, while drainage could not be removed until less than $50 \mathrm{~mL}$ in several days in the ODLP group. Moreover, pain control was another factor influenced hospital stay, pain relief was obviously faster in MA-EMPL group because of the less invasive technique.

\section{Limitations}

There are some limitations in this study. Firstly, the number of included patients in this single-center study is insufficient and may increase data bias. Secondly, cases with different segments involved had not been analyzed, this may increase clinical heterogeneity. And the potential associations between MCSM and spinal cord injury should be further explored, as if the risk of spinal cord injury can be altered by surgical intervention remains unclear (29). Thirdly, the atrophy rate of cervical posterior extensor muscles should be investigated by MRI before and after surgery as it might be involved in clinical outcomes. Moreover, a follow-up of one year is relative short in this study. Hence, a largesample study with long follow-up is required in future study.

\section{Conclusions}

MA-EMPL is an effective, safe and minimally invasive method in treatment of MCSM due to the advantages of maintaining cervical physiological structure as much as possible, improving neurofunction and less related complications. Compared with ODLP, MA-EMPL has advantages in reducing intraoperative blood loss, hospital 
stay, postoperative VAS and axial symptom, as well as preserving postoperative ROM.

\section{Acknowledgments}

We thank the reviewers and editors for their helpful comments on this article, and thank Sun Kyu Choi, a biostatistician working at Yonsei University College of Medicine, for his statistical suggestions.

Funding: The present study was financially supported by the grants of National Natural and Science Foundation of China (81860473), China Scholarship Council (20173109/201708260068), 5511 Innovation-driven Program of Jiangxi Province Department of Science and Technology (20165BCB18017) and Program of Health Commission of Jiangxi Province (20191029, 20155110).

\section{Footnote}

Conflicts of Interest: The authors have no conflicts of interest to declare.

Ethical Statement: The authors are accountable for all aspects of the work in ensuring that questions related to the accuracy or integrity of any part of the work are appropriately investigated and resolved. The study was approved by the Ethical Institutional Review Board of the Second Affiliated Hospital of Nanchang University (2013025) and written informed consents were obtained from all patients.

\section{References}

1. Bakhsheshian J, Mehta VA, Liu JC. Current diagnosis and management of cervical spondylotic myelopathy. Global Spine J 2017;7:572-86.

2. Zhu B, Xu Y, Liu X, et al. Anterior approach versus posterior approach for the treatment of multilevel cervical spondylotic myelopathy: a systemic review and metaanalysis. Eur Spine J 2013;22:1583-93.

3. Luo J, Cao K, Huang S, et al. Comparison of anterior approach versus posterior approach for the treatment of multilevel cervical spondylotic myelopathy. Eur Spine J 2015;24:1621-30.

4. Zhang J, Liu H, Bou EH, et al. Comparative study between anterior cervical discectomy and fusion with ROI-C cage and laminoplasty for multilevel cervical spondylotic myelopathy without spinal stenosis. World
Neurosurg 2019;121:e917-24.

5. Orr RD, Zdeblick TA. Cervical spondylotic myelopathy. Approaches to surgical treatment. Clin Orthop Relat Res 1999;(359):58-66.

6. Arantes Júnior AA, Silva Junior GA, Malheiros JA, et al. A new expansive two-open-doors laminoplasty for multilevel cervical spondylotic myelopathy: technical report and follow-up results. Arq Neuropsiquiatr 2014;72:49-54.

7. Li Z, Xue Y, He D, et al. Extensive laminectomy for multilevel cervical stenosis with ligamentum flavum hypertrophy: more than 10 years follow-up. Eur Spine J 2015;24:1605-12.

8. Yoshida M, Otani K, Shibasaki K, et al. Expansive laminoplasty with reattachment of spinous process and extensor musculature for cervical myelopathy. Spine 1992;17:491-7.

9. Kawaguchi $\mathrm{Y}$, Matsui $\mathrm{H}$, Ishihara $\mathrm{H}$, et al. Axial symptoms after en bloc cervical laminoplasty. J Spinal Disord 1999;12:392-5.

10. Straker L, Skoss R, Burnett A, et al. Effect of visual display height on modeled upper and lower cervical gravitational moment, muscle capacity and relative strain. Ergonomics 2009;52:204-21.

11. Nori S, Iwanami A, Yasuda A, et al. Risk factor analysis of kyphotic malalignment after cervical intramedullary tumor resection in adults. J Neurosurg Spine 2017;27:518-27.

12. Nori S, Shiraishi T, Aoyama R, et al. Muscle-preserving selective laminectomy maintained the compensatory mechanism of cervical lordosis after surgery. Spine (PhilaPa 1976) 2018;43:542-9.

13. Liu X, Chen Y, Yang H, et al. Expansive open-door laminoplasty versus laminectomy and instrumented fusion for cases with cervical ossification of the posterior longitudinal ligament and straight lordosis. Eur Spine J 2017;26:1173-80.

14. Yang F, Li WX, Liu Z, et al. Balance chiropractic therapy for cervical spondylotic radiculopathy: study protocol for a randomized controlled trial. Trials 2016;17:513.

15. Hirabayashi K, Watanabe K, Wakano K, et al. Expansive open-door laminoplasty for cervical spinal stenotic myelopathy. Spine 1983;8:693-9.

16. ItokiK, Kurokawa R, Shingo T, et al. Effect of myoarchitectonic spinolaminoplasty on concurrent hypertension in patients with cervical spondylotic myelopathy. Neurospine 2018;15:77-85.

17. Blizzard DJ, Caputo AM, Sheets CZ, et al. Laminoplasty versus laminectomy with fusion for the treatment of spondylotic cervical myelopathy: short-term follow-up. 
Eur Spine J 2017;26:85-93.

18. Bartels RH, van Tulder MW, Moojen WA, et al. Laminoplasty and laminectomy for cervical spondylotic myelopathy: a systematic review. Eur Spine J 2015;24 Suppl 2:160-7.

19. Vernon H, Mior S. The Neck Disability Index: a study of reliability and validity. J Manipulative Physiol Ther 1991;14:409-15.

20. Nakashima H, Kato F, Yukawa Y, et al. Comparative effectiveness of open-door laminoplasty versus Frenchdoor laminoplasty in cervical compressive myelopathy. Spine (Phila Pa 1976) 2014;39:642-7.

21. Lee DG, Lee SH, Park SJ, et al. Comparison of surgical outcomes after cervical laminoplasty: open-door technique versus French-door technique. J Spinal Disord Tech 2013;26:E198-203.

22. Hukuda S, Ogata M, Mochizuki T, et al. Laminectomy versus laminoplasty for cervical myelopathy: brief report. J Bone Joint Surg Br 1988;70:325-6.

23. Takeuchi K, Yokoyama T, Aburakawa S, et al. Axial symptoms after cervical laminoplasty with C3 laminectomy compared with conventional C3-C7 laminoplasty: a modified laminoplasty preserving the semispinalis cervicis inserted into axis. Spine (Phila Pa 1976) 2005;30:2544-9.

24. Tsuji T, Matsumoto M, Nakamura M, et al. Factors associated with postoperative C5 palsy after expansive open-door laminoplasty: retrospective cohort study using multivariable analysis. Eur Spine J 2017;26:2410-6.

25. Lau D, Winkler EA, Than KD, et al. Laminoplasty versus laminectomy with posterior spinal fusion for multilevel cervical spondylotic myelopathy: influence of cervical alignment on outcomes. J Neurosurg Spine 2017;27:508-17.

26. Meng Y, Wang X, Chen H, et al. Risk factors for significant intraoperative blood loss during unilateral expansive open-door cervical laminoplasty for cervical compressive myelopathy. World Neurosurg 2018;114:e1253-60.

27. Rodriguez-Feo JA, Leas D, Odum SM, et al. Reoperation rates following open-door cervical laminoplasty. Int J Spine Surg 2018;12:751-6.

28. Phan K, Mobbs RJ. Minimally invasive versus open laminectomy for lumbar stenosis: a systematic review and Meta-analysis. Spine (Phila Pa 1976) 2016;41:E91-100.

29. Wu JC, Chen YC, Huang WC. Ossification of the posterior longitudinal ligamentin cervical spine: prevalence, management, and prognosis. Neurospine 2018;15:33-41.
Cite this article as: Yu Z, He D, Xiong J, Pan Z, Feng L, Xu J, Han Z, Gragnaniello C, Koga H, Phan K, Azimi P, Lee JJ, Ha Y, Cao K. Extensor muscle-preserving laminectomy in treating multilevel cervical spondylotic myelopathy compared with laminoplasty. Ann Transl Med 2019;7(18):472. doi: 10.21037/ atm.2019.08.17 